OPEN ACCESS

Edited by:

Kyung Hyun Sung,

UCLA Health System, United States

Reviewed by: Nguyen Minh Duc,

Pham Ngoc Thach University of Medicine, Vietnam

Zheng Han,

Johns Hopkins University,

United States

*Correspondence:

Dehong Luo

13926236152@163.com

Yin Wu

yin.wu@siat.ac.cn

${ }^{\dagger}$ These authors have contributed equally to this work and share first authorship

Specialty section: This article was submitted to Cancer Imaging and Image-directed Interventions, a section of the journal

Frontiers in Oncology

Received: 26 November 2021 Accepted: 14 January 2022

Published: 08 February 2022

Citation:

Liu Z, Zou L, Yang Q, Qian L, Li T, Luo H, Che C, Lei Y, Chen P, Qiu C, Liu X, Wu Y and Luo D (2022) Baseline Amide Proton Transfer Imaging at $3 T$

Fails to Predict Early Response to Induction Chemotherapy in Nasopharyngeal Carcinoma.

Front. Oncol. 12:822756. doi: 10.3389/fonc.2022.822756

\section{Baseline Amide Proton Transfer Imaging at 3T Fails to Predict Early Response to Induction Chemotherapy in Nasopharyngeal Carcinoma}

\author{
Zhou Liu ${ }^{1,2 \dagger}$, Liyan Zou ${ }^{1 \dagger}$, Qian Yang ${ }^{1}$, Long Qian ${ }^{3}$, Tianran Li $^{1}$, Honghong Luo ${ }^{1}$, \\ Canwen Che ${ }^{1}$, Yuanyuan Lei ${ }^{4}$, Peng Chen ${ }^{5}$, Chunyan Qiu ${ }^{5}$, Xin Liu ${ }^{2}$, \\ Yin $\mathrm{Wu}^{2 *}$ and Dehong Luo ${ }^{1,6 *}$
}

${ }^{1}$ Department of Radiology, National Cancer Center/National Clinical Research Center for Cancer/Cancer Hospital \& Shenzhen Hospital, Chinese Academy of Medical Sciences and Peking Union Medical College, Shenzhen, China, 2 Paul C. Lauterbur Research Center for Biomedical Imaging, Shenzhen Institute of Advanced Technology, Chinese Academy of Sciences, Shenzhen, China, ${ }^{3}$ MR Research, GE Healthcare, Beijing, China, ${ }^{4}$ Department of Pathology, National Cancer Center/National Clinical Research Center for Cancer/Cancer Hospital \& Shenzhen Hospital, Chinese Academy of Medical Sciences and Peking Union Medical College, Shenzhen, China, ${ }^{5}$ Department of Radiation Oncology, National Cancer Center/ National Clinical Research Center for Cancer/Cancer Hospital \& Shenzhen Hospital, Chinese Academy of Medical Sciences and Peking Union Medical College, Shenzhen, China, ${ }^{6}$ Department of Radiology, National Cancer Center/National Clinical Research Center for Cancer/Cancer Hospital, Chinese Academy of Medical Sciences and Peking Union Medical College, Beijing, China

Background: Early identification of nasopharyngeal carcinoma (NPC) patients with high risk of failure to induction chemotherapy (IC) would facilitate prompt individualized treatment decisions and thus reduce toxicity and improve overall survival rate. This study aims to investigate the value of amide proton transfer (APT) imaging in predicting short-term response of NPC to IC and its potential correlation with well-established prognosis-related clinical characteristics.

Methods and Materials: A total of 80 pathologically confirmed NPC patients receiving pre-treatment APT imaging at 3T were retrospectively enrolled. Using asymmetry analysis, APT maps were calculated with mean ( $\mathrm{APT}_{\text {mean }}$ ), $90^{\text {th }}$ percentile (APT 90 ) of APT signals in manually segmented NPC measured. APT values were compared among groups with different histopathological subtypes, clinical stages (namely, $\mathrm{T}, \mathrm{M}, \mathrm{N}$, and overall stages), EBV-related indices (EBV-DNA), or responses to induction chemotherapy, using MannWhitney $\mathrm{U}$ test or Kruskal-Wallis $\mathrm{H}$ test.

Results: NPC showed significantly higher $\mathrm{APT}_{\text {mean }}$ than normal nasopharyngeal tissues $(1.81 \pm 0.62 \%$ vs. $1.32 \pm 0.56 \%, P<0.001)$. APT signals showed no significant difference between undifferentiated and differentiated NPC subtypes groups, different EBV-DNA groups, or among T, N, M stages and overall clinical stages of II, III, IVA and IVB (all $P>0.05)$. Similarly, baseline APT-related parameters did not differ significantly among 
different treatment response groups after IC, no matter if evaluated with RECIST criteria or sum volumetric regression ratio (SVRR) (all $P>0.05$ ).

Conclusion: NPC showed significantly stronger APT effect than normal nasopharyngeal tissue, facilitating NPC lesion detection and early identification. However, stationary baseline APT values exhibited no significant correlation with histologic subtypes, clinical stages and EBV-related indices, and showed limited value to predict short-term treatment response to IC.

Keywords: amide proton transfer (APT), nasopharyngeal carcinoma (NPC), Epstein-Barr virus (EBV), clinical stage, induction chemotherapy

\section{INTRODUCTION}

The number of the newly diagnosed nasopharyngeal carcinoma (NPC) patients has been reported to be over 129,000 worldwide in 2018, among which $70 \%$ is aggregated in East Asia and Southeast Asia (1). In particular, the incidence is as high as 9.69/100,000 in Southeastern China (2). Currently, induction chemotherapy (IC) followed by concurrent chemoradiotherapy (CCRT) has become the standard treatment for locoregionally NPC for its better prognosis than CCRT alone $(3,4)$. However, around $10 \%$ patients still do not respond well to IC. Identification of those non-responders in advance would facilitate more prompt personalized treatment decisions, avoiding chemotherapy toxicity and unnecessary costs. In addition, tumor response to IC has been identified as an independent prognostic factor for long-term survival $(5,6)$. Hence, pretreatment prediction of short-term response to IC is essential for better individualized management of NPC patients.

Due to substantial biological heterogeneity, NPCs with the same clinical stage might have completely different responses to IC. Identification of biomarkers that could reflect tumor microenvironment characteristics may benefit the prediction of treatment response. With the advancement of morphological and function imaging techniques, MRI has been widely used for NPC evaluation by providing information at both macroscopic and microscopic levels (7-10). Recently, APT-weighted (APT $\left.{ }_{w}\right)$ imaging has emerged as a powerful tool to probe the chemical exchange saturation transfer (CEST) effect between endogenous proteins/peptide amide protons and bulk water (11). Because of overexpressed proteins in tumors, $\mathrm{APT}_{\mathrm{w}}$ imaging has been increasingly used in tumor detection, grading, and treatment response evaluation of various types of cancer (12-26). Although $\mathrm{APT}_{\mathrm{w}}$ imaging has shown promising efficacy in predicting middleterm outcome after complete standard treatment (induction

\footnotetext{
Abbreviations: NPC, nasopharyngeal carcinoma; APT, amide proton transfer; CEST, chemical exchange saturation transfer; EBV, Epstein-Barr virus; AJCC, American Joint Committee on Cancer; WASSR, water saturation shift referencing; $\mathrm{APT}_{\text {mean }}$, Mean value of APT asymmetry; $\mathrm{APT}_{90}$, 90th percentile of APT asymmetry; RECIST, Response Evaluation Criteria in Solid Tumors; SVRR, sum volumetric regression ratio; EBER, EBV-encoded small RNA; ISH, in situ hybridization; EBV-DNA, Epstein-Barr deoxyribonucleic acid; EA-IgA, early antigen antibody immunoglobulin A; VCA-IgA, viral capsid antibody immunoglobulin A; MTR, magnetization transfer ratio.
}

chemotherapy followed by concurrent chemoradiotherapy) (26) or 2-year long-term outcome prediction in NPC patients (27), little is known about whether APT signal could predict short-term treatment response to IC only.

Besides, NPC can be classified into distinct subtypes by wellestablished prognosis-related characteristics, e.g. Epstein-Barr virus (EBV) infection status (28), different histopathological subtypes (29) and clinical stages, etc. It remains unknown whether these distinct NPC subtypes with different prognosis have different protein metabolism levels, which potentially could be quantified by $\mathrm{APT}_{\mathrm{w}}$ imaging. Therefore, if there is any association between baseline APT measurements and wellestablished prognosis-related clinical characteristics of NPC, such as histopathological subtypes, clinical stages, and status of Epstein-Barr (EB) virus infection, the value of APT in predicting prognosis would be further strengthened.

In this study, we aimed to investigate the potential value of $\mathrm{APT}_{\mathrm{w}}$ imaging in predicting short-term response to IC and its potential association with prognosis-related histopathological subtypes, clinical stages, and EBV-related indices.

\section{MATERIALS AND METHODS}

\section{Patient Enrollment}

This retrospective study was approved by the local ethics committee of the Cancer Hospital \& Shenzhen Hospital, Chinese Academy of Medical Sciences, and informed consent was waived. From December 2019 to August 2021, 114 patients with suspected NPC who had undergone baseline APT-weighed imaging were enrolled for further selection. The inclusion and exclusion criteria were as follows: 1) biopsy confirmed NPC; 2) the maximal diameter of the primary nasopharyngeal lesion was larger than $1 \mathrm{~cm}$; 3) conventional MRI and APT-weighted imaging were performed before treatment; 4) each patient had received at least one examination for clinical staging work-up, such as chest X-ray, abdominal ultrasonography, chest and abdomen CT, bone SPECT scan, or whole-body PET-CT scan. Clinical staging for each NPC patient was performed based on the 8th edition of the American Joint Committee on Cancer (AJCC) (30); APT-weighted images were reviewed by a boardcertified radiologist (ZL with more than 10 years of experience in head and neck imaging) and those with degraded image quality, 
namely, overt signal lost, motion artifacts, geometric distortion, were excluded from analysis.

\section{Treatment Strategy}

All NPC patients received standard treatment. For NPC patients with clinical stages of I and II, concurrent chemoradiotherapy or radiotherapy alone was given, whereas IC followed by concurrent chemoradiotherapy were prescribed to patients with clinical stages of III and IV. The brief treatment procedure was: 1) IC (GP regimen): Gemcitabine $\left(1 \mathrm{~g} / \mathrm{m}^{2}\right.$, Days 1 and 8$)+$ cisplatin $\left(75 \mathrm{mg} / \mathrm{m}^{2}\right.$, Day 1); or Paclitaxel $\left(135-175 \mathrm{mg} / \mathrm{m}^{2}\right.$, Day 1) + cisplatin $\left(75 \mathrm{mg} / \mathrm{m}^{2}\right.$, Day 1$), 3$ weeks per cycle $\times 2-3$ cycles; 2$)$ Concurrent chemoradiotherapy: cisplatin $\left(30 \mathrm{mg} / \mathrm{m}^{2}\right.$, Day 1 , once a week for 7 times, iv) and concurrent radiation therapy (in a total of 6.6-7.6 weeks, accumulated dose of 66-78 Gy for primary lesion, while 60-70 Gy for regional lymph nodes). With intensity-modulated radiation therapy being used, conventional $6 \mathrm{MV}-\mathrm{X}$ fractionated radiotherapy $(200 \mathrm{cGy}$ each time $\times 1$ time per day $\times 5$ days per week) was performed, which covers the primary NPC lesion and bilateral cervical lymphatics at risk.

\section{Follow-Up and Study Endpoint}

In this study, the endpoint was set between after IC completion and before next-step concurrent chemoradiotherapy. Standard clinical MRI follow-up was performed at the endpoint for each subject.

\section{MRI Study}

MRI study was performed on a 3T MR scanner (Discovery MR 750w, General Electric) using a 32-channel head and neck joint coil. Local shimming was placed to cover the whole nasopharyngeal carcinoma to reduce static magnetic field inhomogeneity. Referencing to T2-weighted images, a singleslice axial plane covering the largest primary nasopharyngeal carcinoma was selected for APT imaging with chemical shiftselective fat suppression and single-shot fast spin echo (FSE) readout (4 RF saturation pulses with duration of $500 \mathrm{~ms}$ for each pulse and without interpulse delay, RF saturation power $B_{1}$ of 2 $\mu \mathrm{T}, \mathrm{TR}=6.5 \mathrm{~s}$ with minimum $\mathrm{TE}$, slice thickness $=4 \mathrm{~mm}$, field of view $=22 \times 22 \mathrm{~cm}^{2}$, matrix $=128 \times 128$ ). In additional to an unsaturated scan, 27 CEST-weighted images with frequency offsets of $0, \pm 0.5, \pm 1.0, \pm 1.5, \pm 2.0, \pm 2.5, \pm 3.0, \pm 3.5, \pm$ $4.5, \pm 5.0, \pm 5.5, \pm 6.0, \pm 6.5$, and $\pm 7.0 \mathrm{ppm}$ were acquired. The total acquisition time is approximately $4.5 \mathrm{~min}$. A water saturation shift referencing (WASSR) map was collected with $\mathrm{B}_{1}$ of $0.5 \mu \mathrm{T}$ (frequency offsets between $\pm 240 \mathrm{~Hz}$ with intervals of $48 \mathrm{~Hz}$ ) to correct $\mathrm{B}_{0}$ inhomogeneity.

\section{Data Analysis}

APT data was analyzed in MATLAB (MathWorks). Z-spectra (S) was normalized by the unsaturated scan $\left(\mathrm{S}_{0}\right)$, interpolated by smoothing splines, and corrected the field inhomogeneity with WASSR $(31,32)$. Pixel-wise APT-weighted value was calculated using asymmetry analysis of $\mathrm{APT}_{\mathrm{w}}$ (APT-weighted) $=\mathrm{S}(-3.5$ $\mathrm{ppm}) / \mathrm{S}_{0}-\mathrm{S}(3.5 \mathrm{ppm}) / \mathrm{S}_{0}$.

Region of interest (ROI) of primary NPC was manually delineated on the unsaturated image by a radiologist (LZ with
6 years of experience in head and neck imaging) using opensource ITK-SNAP software (http://itksnap.org) with referencing conventional anatomical images, namely, T1-weighted, T2weighted, and contrast enhanced T1-weighted images. Overt hemorrhage, air space, necrosis and vessels were excluded. For patients with tumor confined in one side of nasopharynx without involving the midline of posterior wall of nasopharynx, the normal nasopharyngeal tissue was delineated as well. ROI delineation was then reviewed and approved by a senior radiologist (ZL with more than 10 years of experience in head and neck imaging). $\mathrm{APT}_{\mathrm{w}}$ signals, namely, the average $\mathrm{APT}$ $\left(\mathrm{APT}_{\text {mean }}\right)$ and $90^{\text {th }}$ percentile $\left(\mathrm{APT}_{90}\right)$, were obtained from each ROI.

\section{Treatment Response Evaluation}

The largest diameter of NPC was manually measured on the slice with the largest area of lesion on axial $\mathrm{T}_{1}$-weighted contrast enhanced images by a board-certified radiologist (LZ with 6 years of experience in head and neck imaging) on the vendor workstation. The measurement was reviewed by a senior radiologist (ZL), with any discrepancies resolved through consensus discussion. Then, the treatment response of primary tumor was determined by calculating the relative change percentage of tumor maximal diameter between pre- and posttreatment, as $\Delta \mathrm{D}=\left(\mathrm{D}_{\text {pre-treatment }}-\mathrm{D}_{\text {post-treatment }}\right) / \mathrm{D}_{\text {pre-treatment }}$, and classified as complete response ( $\mathrm{CR}, \Delta \mathrm{D}=100 \%)$, partial response ( $\mathrm{PR}, \Delta \mathrm{D} \geq 30 \%$ ), stable disease ( $\mathrm{SD},-20 \%<\Delta \mathrm{D}<30 \%$ ), and progression disease ( $\mathrm{PD}, \Delta \mathrm{D} \leq-20 \%)$ according to the RECIST criteria version 1.1 (33).

Tumor volume was measured by manually delineating and summing NPC lesion from consecutive 2D slices on contrastenhanced $\mathrm{T}_{1}$-weighted image by $\mathrm{LZ}$ and then reviewed by a senior radiologist $(\mathrm{ZL})$. The sum volumetric regression ratio (SVRR) was defined as the tumor volume reduction percentage before and after treatment, and 50\% reduction in SVRR was arbitrarily chosen as the cut-off value.

\section{Histopathology and Epstein-Barr (EB) Virus-Related Indices}

The following pathological information were collected: 1) histology of nasopharyngeal carcinoma based on $\mathrm{WHO}$ classification, namely, nonkeratinizing squamous cell carcinoma (differentiated subtype and undifferentiated subtype), keratinizing squamous cell carcinoma and basaloid squamous cell carcinoma (34); 2) EBV-encoded small RNA (EBER) status using in situ hybridization (ISH). Serum test reports before treatment were also examined to obtain the following information: 1) copy numbers of viral Epstein-Barr deoxyribonucleic acid (EBV-DNA) in plasma samples before treatment, whereas copy numbers of 400 as threshold; 2) serum level of early antigen antibody (EA-IgA); and 3) serum level of viral capsid antibody (VCA-IgA).

\section{Statistical Analysis}

All the statistical analyses were performed using SPSS software v. 26.0 (IBM, Armonk, NY). Paired Student's t-test was performed to evaluate the difference in $\mathrm{APT}_{\mathrm{w}}$ signals between normal and 
cancerous nasopharyngeal tissues. Continuous variables were displayed as median (25th-75th percentile). Mann-Whitney $U$ test was used between the two histopathological subtypes. Kruskal-Wallis $\mathrm{H}$ test was conducted to assess any significant differences among groups with different clinical stages or treatment responses. Receiver operating characteristic (ROC) analysis was performed and the area under the curve (AUC) was calculated. The optimal cutoff values were determined by maximizing the sum of sensitivity and specificity using YOUDEN index. Pearson's correlation analysis was used to evaluate the relationship between $\mathrm{APT}_{\mathrm{w}}$ measurements and tumor regression rate.

\section{RESULTS}

A total of 80 patients were included in the analysis (Figure 1) with clinical characteristics summarized in Table $\mathbf{1}$.

\section{Tissue Characterization}

A total of 17 patients showed NPC confined in one side and not involving the midline of nasopharynx. Figures 2A, B illustrate $\mathrm{APT}_{\mathrm{w}}$ maps and histograms in NPC and normal nasopharyngeal tissue regions in a 66-year-old male patient. The $\mathrm{APT}_{\text {mean }}$ of the NPC were significantly higher than that of corresponding normal nasopharyngeal tissue $(1.81 \pm 0.62 \%$ vs. $1.32 \pm 0.56 \%, P<0.001)$ (Figure 2C). However, $\mathrm{APT}_{90}$ showed no significant difference between NPC and corresponding normal nasopharyngeal tissue $(3.68 \pm 0.84 \%$ vs. $3.25 \pm 1.07 \%, P=0.115)$. The optimal cutoff value of $\mathrm{APT}_{\text {mean }}$ in discriminating NPC with contralateral nasopharyngeal tissue was $1.66 \%$ with a sensitivity of 0.765 , a specificity of 0.765 , and an AUC of 0.727 (Figure 2D).

Among the enrolled 80 patients, 66 had undifferentiated carcinoma, 14 had differentiated carcinoma, and no patients had nonkeratinizing squamous cell carcinoma. All $\mathrm{APT}_{\mathrm{w}}$ signals did not differ significantly between undifferentiated and differentiated NPC groups (all $P>0.05$ ) (Figure 3 ), as summarized in Table 2.

\section{TNM Stage}

No significant difference was found in $\mathrm{APT}_{\mathrm{w}}$ signals among primary tumor stages of T1 to T4, among lymph node stages of N1 to N3 (Figure 4), between M0 and M1 stages, among overall clinical stages of II, III, IVA, and IVB (all $P>0.05$ ) (Table 2).

\section{EBV-Related Indices}

The 67 patients with available EBER status all showed positive EBER on the specimen using in situ hybridization in all cases. For the 76 cases with available serum EBV-DNA quantification, $\mathrm{APT}_{\mathrm{w}}$ signals was found to be comparable between EBV-DNA positive group and EBV-DNA negative group (Figure 5). Similarly, there was no significant difference in $\mathrm{APT}_{\mathrm{w}}$ signals between EA-IgA positive group $(\mathrm{N}=18)$ and EA-IgA negative group $(\mathrm{N}=16)$, and between VCA-IgA positive group $(\mathrm{N}=27)$ and VCA-IgA negative group $(\mathrm{N}=7)($ all $P>0.05)$ for the 34 NPC patients with available serum expression of EBV antibody (Table 3).

\section{Treatment Response}

Among 66 patients with available treatment response evaluation after overall treatment, 53 patients had received induction chemotherapy (Table 4). For patients receiving overall treatment, no significant correlation was found neither between $\mathrm{APT}_{\text {mean }}$ and $\operatorname{SVRR}\left(\mathrm{R}^{2}=-0.014, P=0.738\right)$ (Figure 6A) nor between $\mathrm{APT}_{90}$ and $\operatorname{SVRR}\left(\mathrm{R}^{2}=-0.001, P=\right.$ 0.339) (Figure 6B). Similarly, for patients who received induction chemotherapy, SVRR did not significantly correlate with neither $\mathrm{APT}_{\text {mean }}\left(\mathrm{R}^{2}=-0.019, P=0.883\right)$ (Figure 6C), nor $\mathrm{APT}_{90}\left(\mathrm{R}^{2}=0.008, P=0.238\right)$ (Figure 6D). Baseline APT-related parameters did not differ significantly among different treatment

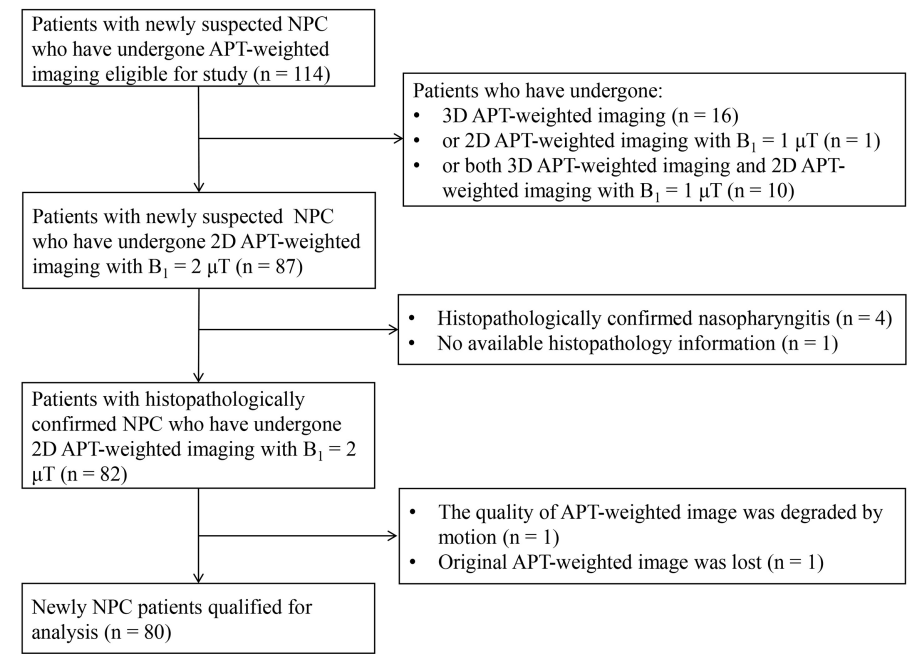

FIGURE 1 | Flowchart of patient enrollment. 
TABLE 1 | Clinical characteristics of the enrolled 80 patients.

\begin{tabular}{|c|c|}
\hline Clinical characteristics & $\begin{array}{l}\text { Values/No. of } \\
\text { patients }\end{array}$ \\
\hline Age (years) & $\begin{array}{c}41.5 \\
(34-51.25)\end{array}$ \\
\hline \multicolumn{2}{|l|}{ Gender } \\
\hline Male & 55 \\
\hline Female & 25 \\
\hline \multicolumn{2}{|l|}{ Histology } \\
\hline Nonkeratinizing squamous cell carcinoma & 0 \\
\hline Undifferentiated carcinoma & 66 \\
\hline Differentiated carcinoma & 14 \\
\hline \multicolumn{2}{|l|}{ Primary tumor stage } \\
\hline $\mathrm{T} 1$ & 16 \\
\hline $\mathrm{T} 2$ & 8 \\
\hline Т3 & 41 \\
\hline T4 & 15 \\
\hline \multicolumn{2}{|l|}{ Nodal stage } \\
\hline NO & 1 \\
\hline $\mathrm{N} 1$ & 25 \\
\hline $\mathrm{N} 2$ & 25 \\
\hline N3 & 29 \\
\hline \multicolumn{2}{|l|}{ Metastatic stage } \\
\hline MO & 66 \\
\hline M1 & 11 \\
\hline $\mathrm{Mx}$ & 3 \\
\hline \multicolumn{2}{|l|}{ Clinical stage } \\
\hline 1 & 0 \\
\hline$\|$ & 5 \\
\hline III & 33 \\
\hline IVA & 27 \\
\hline IVB & 12 \\
\hline Not available & 3 \\
\hline \multicolumn{2}{|l|}{ Treatment strategy } \\
\hline Radiotherapy only & 1 \\
\hline Concurrent chemoradiotherapy & 15 \\
\hline Induction chemotherapy and concurrent chemoradiotherapy & 58 \\
\hline Treatment strategy not available & 6 \\
\hline \multicolumn{2}{|l|}{ MRI scan } \\
\hline Baseline MR scan & 80 \\
\hline Follow-up MR scan after induction chemotherapy & 53 \\
\hline Follow-up MR scan after radiotherapy only & 1 \\
\hline $\begin{array}{l}\text { Follow-up MR scan after concurrent } \\
\text { chemoradiotherapy }\end{array}$ & 12 \\
\hline No follow-up MR scan & 14 \\
\hline
\end{tabular}

response groups after overall treatment or after induction chemotherapy, no matter evaluated with the RECIST criteria or SVRR (all $P>0.05$ ). Besides, no significant difference in tumor regression rate was found between EBV-DNA $(+)(n=16)$ and EBV-DNA $(-)(\mathrm{n}=37)$ group who received induction chemotherapy, as evaluated with the RECIST criteria (52.0 (38.3-57.2)\% vs.58.0 (35.0-100.0)\%, $P=0.407)$ and SVRR (67.0 (52.5-88)\% vs. $79.0(56.0-100.0) \%, P=0.441)$, respectively.

\section{DISCUSSION}

In this study, we investigated the value of pre-treatment $\mathrm{APT}_{\mathrm{w}}$ signal in predicting short-term response after IC in NPC patients and its potential association with prognosis-related clinical characteristics, namely, histopathological subtypes, clinical stages, and EBV-related indices. Unfortunately, based on current result, the stationary baseline APT-weighted imaging showed limited value to predict response to IC and no significant correlation with different histopathological subtypes, EBV infection statuses and clinical stages.

The nasopharynx APT MRI is challenging due to susceptibility artifacts, and swallowing and jaw motion (22, 35). Our image quality was visually comparable to those shown in previous APT studies $(22,35)$ without apparent signal loss, geometric distortion, or any artifacts. In this study, significantly higher mean APT value in NPC tissue was observed than that in normal nasopharyngeal tissue, which could facilitate NPC lesion detection and early identification. The stronger APT effect may reflect rapidly proliferating tumor cells and abnormally active proteo-synthesis in cancerous tissues, as postulated previously (36).

Although APT-weighted imaging has been proven to be useful in differentiating head and neck malignant and benign tumors $(22,23)$, it was reported to be difficult in distinguishing malignant tumor types in head and neck regions (23). Our study showed that $\mathrm{APT}_{\mathrm{w}}$ signals failed to discriminate between undifferentiated and differentiated NPC histology subtypes, which may imply similar protein synthesis status of these two histology subtypes before treatment. As for clinical stages, no significant difference of $\mathrm{APT}_{\mathrm{w}}$ signals was shown among $\mathrm{T}, \mathrm{N}$, and $M$ stages, and overall clinical stages.

EB-virus infection was recognized as the most common etiology of NPC, especially for nasopharyngeal non-keratinizing squamous cell carcinoma in endemic area (37). Consistently, all cases with available EBER in situ hybridization were tested EBER positive in our study. The serum EBV-DNA copy number has already been widely used to screen population with the high risk of developing NPC in endemic areas (38) and may serve as a biomarker to monitor and predict treatment response (39). However, pretreatment EBVDNA status does not show apparent relationship with tumor regression rate after IC in this study. In contrast, a multi-center phase II randomized controlled study showed that an early decrease of EBV-DNA copy number indicates better treatment response (40). This might imply that monitoring the dynamic change of EBV-infection status throughout the treatment course using EBVDNA copy number might be more effective than merely detecting a stationary pretreatment status in predicting treatment response. However, EBV-DNA copy number quantification data were not available after treatment in this retrospective study. Besides, although it seems no significant correlation of APT signals with EBV-related indices was presented, the correlation between $\mathrm{APT}_{\mathrm{w}}$ signals change and EBV-DNA copy number alteration should be investigated in the future.

This study focused on the investigation of the value of APT imaging in predicting short-term survival of NPC patients. Although Qamar et al. $(26,27)$ demonstrated that the mean APT value change between pretreatment and intra-treatment 2 weeks later indeed could predict the middle-term outcome six months after the completion of standard treatment, both pretreatment and intra-treatment mean APT value did not 

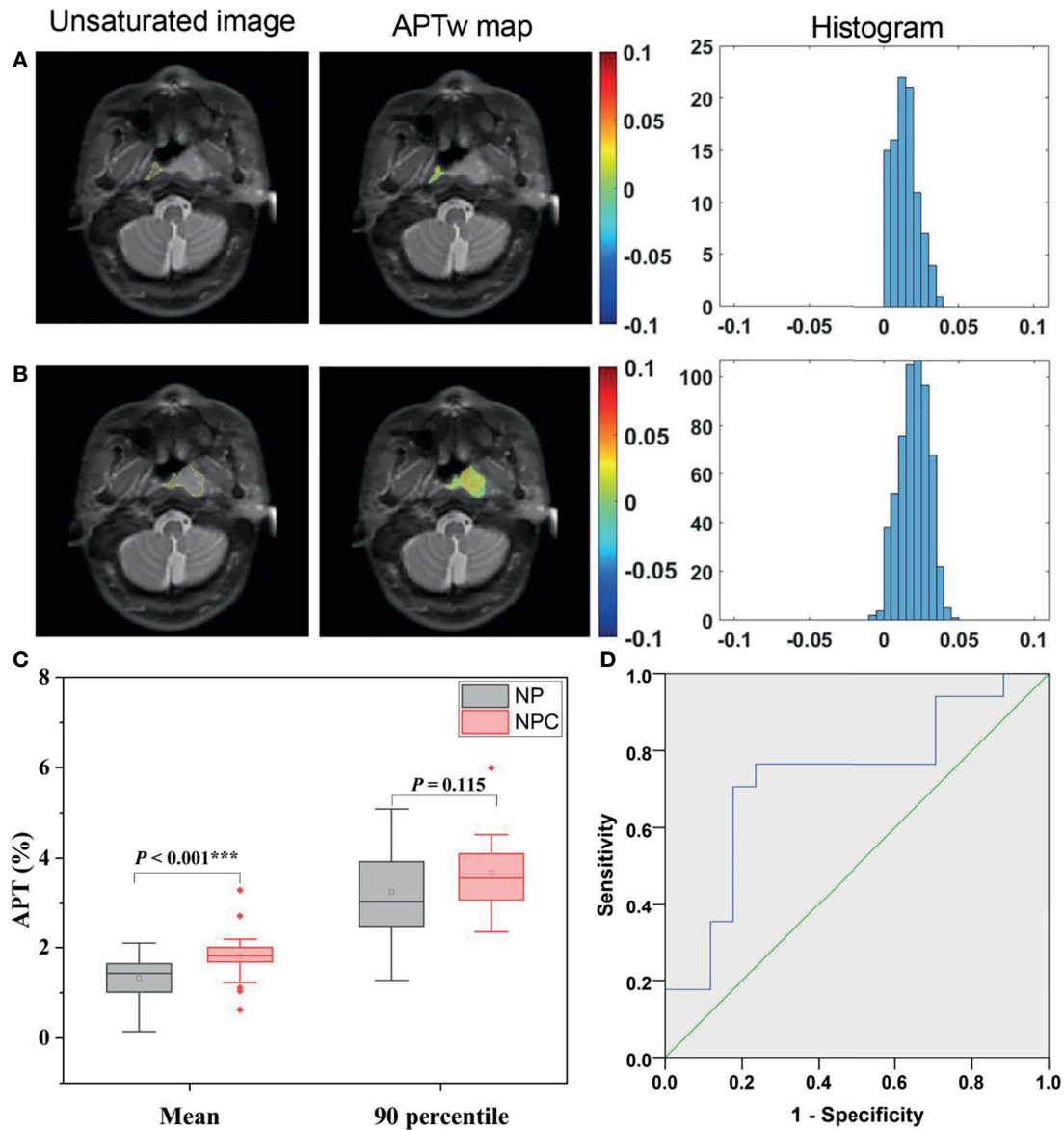

FIGURE 2 | Representative ROI delineation on unsaturated SO images, APT $w$ maps, and histograms in NPC (A) and normal nasopharyngeal tissue (B) regions in a 66-year-old male patient. Comparison of $\mathrm{APT}_{w}$ signals, including APT mean, APT 90 (C) between NPC and corresponding normal nasopharynx tissues; ROC analyses of $\mathrm{APT}_{\text {mean }}$ in discriminating NPC and normal nasopharynx tissue (D).

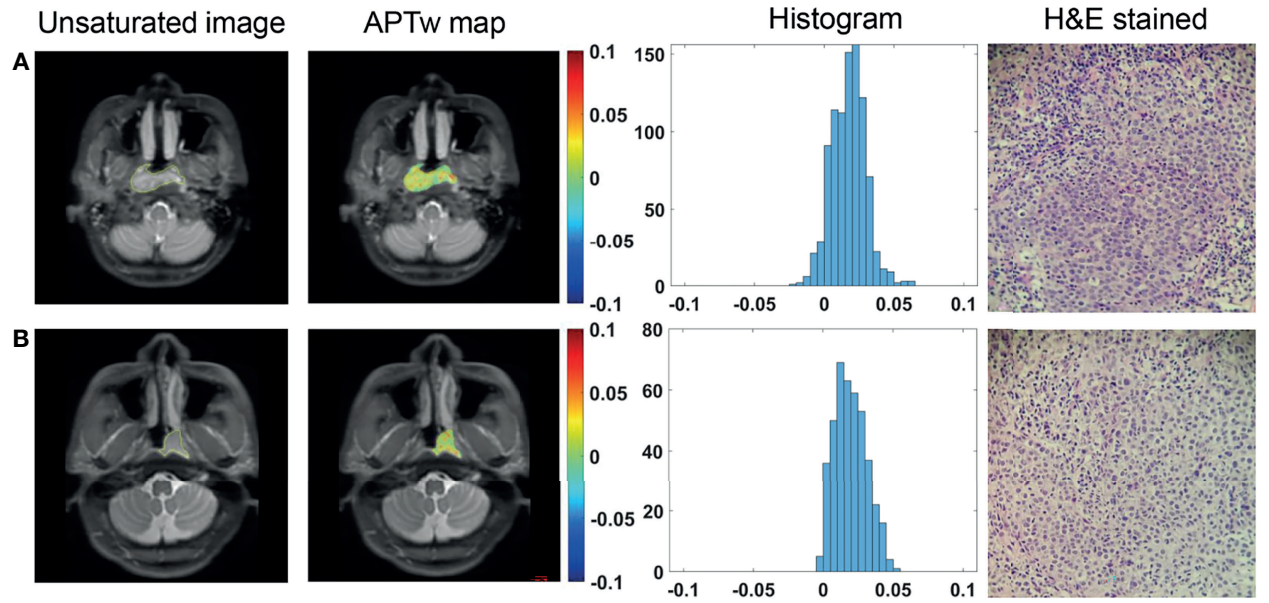

FIGURE 3 | ROI delineation on unsaturated SO images with ROls overlaid, APT $w$ maps and histograms, and H\&E stained slides (40 × 10) of a 34 -year-old female patient with differentiated NPC (A) and a 36-year-old male patient with undifferentiated NPC (B). 
TABLE 2 | APT-related parameters in different histology and TNM stage groups.

\begin{tabular}{|c|c|c|c|}
\hline & No. & $\mathrm{APT}_{\text {mean }}(\%)$ & $\mathrm{APT}_{90}(\%)$ \\
\hline Nonkeratinizing squamous cell carcinoma & 0 & - & - \\
\hline Undifferentiated carcinoma & 66 & $1.72(1.36-2.10)$ & $3.54(3.05-4.04)$ \\
\hline Differentiated carcinoma & 14 & $1.77(1.45-1.99)$ & $3.20(3.14-3.77)$ \\
\hline$P$-value & - & 0.810 & 0.695 \\
\hline \multicolumn{4}{|l|}{ Primary tumor stage } \\
\hline T1 & 16 & $1.81(1.60-2.07)$ & $3.70(3.46-4.11)$ \\
\hline T2 & 8 & $2.01(1.72-2.42)$ & $3.67(3.23-4.52)$ \\
\hline T3 & 41 & $1.64(1.32-1.96)$ & $3.39(2.86-3.74)$ \\
\hline T4 & 15 & $1.80(1.31-2.26)$ & $3.22(3.03-4.16)$ \\
\hline$P$-value & - & 0.330 & 0.103 \\
\hline \multicolumn{4}{|l|}{ Nodal stage } \\
\hline NO & 1 & - & - \\
\hline N1 & 25 & $1.76(1.48-2.00)$ & $3.44(3.14-4.09)$ \\
\hline N2 & 25 & $1.86(1.48-2.14)$ & 3.59 (3.06-4.28) \\
\hline N3 & 29 & $1.72(1.32-2.00)$ & $3.45(3.07-3.82)$ \\
\hline$P$-value & - & 0.644 & 0.867 \\
\hline \multicolumn{4}{|l|}{ Metastatic stage } \\
\hline Mo & 66 & $1.74(1.48-2.01)$ & 3.42 (3.05-4.03) \\
\hline M1 & 11 & $1.53(1.02-2.21)$ & $3.74(3.64-4.05)$ \\
\hline $\mathbf{M x}^{\mathrm{a}}$ & 3 & - & - \\
\hline$P$-value & - & 0.432 & $0.048^{\star}$ \\
\hline \multicolumn{4}{|l|}{ Overall tumor stage } \\
\hline I & 0 & - & - \\
\hline II & 5 & $1.76(1.62-1.80)$ & 3.25 (2.89-4.04) \\
\hline III & 33 & $1.86(1.58-2.14)$ & $3.56(3.14-4.09)$ \\
\hline IVA & 27 & $1.59(1.36-1.91)$ & $3.16(2.95-3.58)$ \\
\hline IVB & 12 & $1.62(1.08-2.18)$ & $3.75(3.67-4.10)$ \\
\hline Not available & 3 & - & - \\
\hline$P$-value & - & 0.343 & 0.068 \\
\hline
\end{tabular}

${ }^{*} P<0.05$ indicates significant difference. $M x^{a}$, metastatic status undetermined.
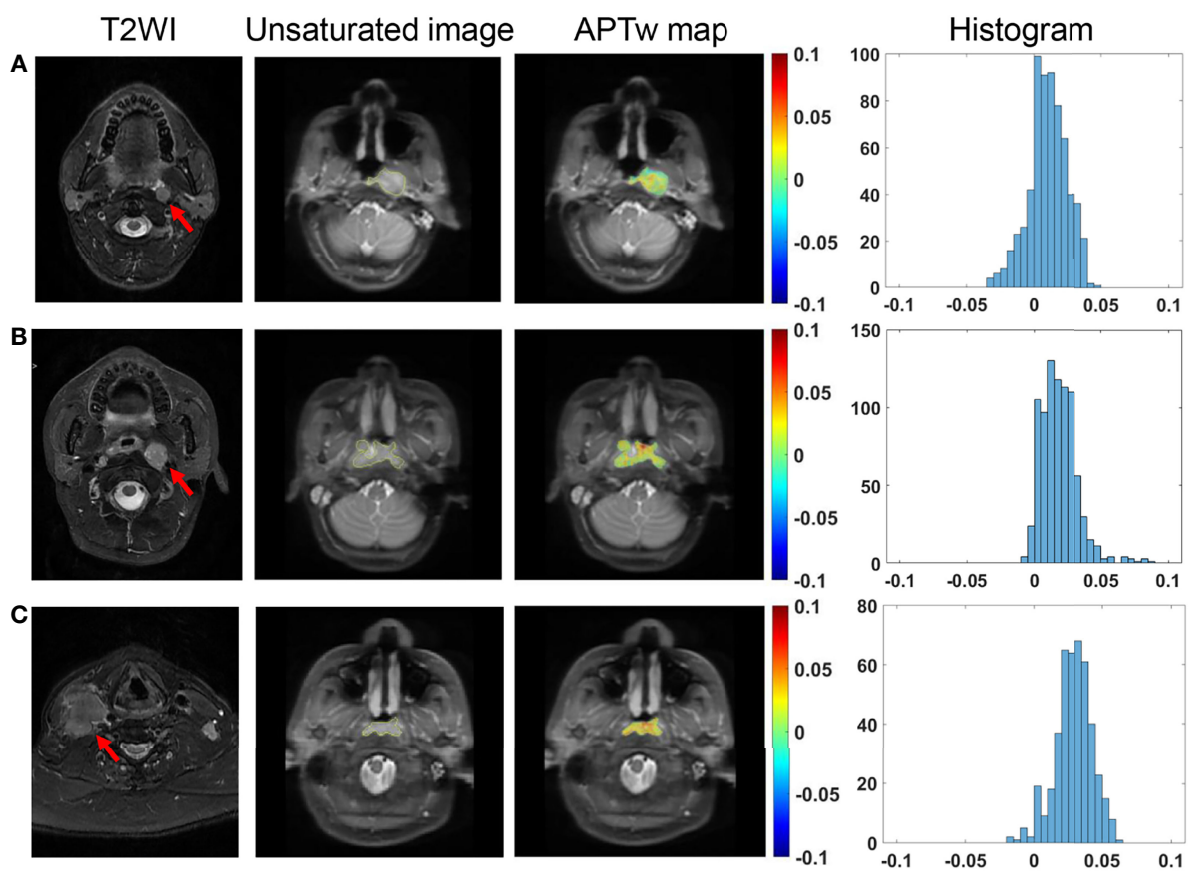

FIGURE 4 | Comparison of T2-weighted images of metastatic lymph nodes (red arrows), and unsaturated SO images with ROI overlaid, APT $w$ maps and histograms in NPC tissues among different N stages. A 39-year-old male patient with N1 stage NPC (A), a 33-year-old female patient with N2 stage NPC (B), and a 65-year-old male patient with N3 stage NPC (C). 

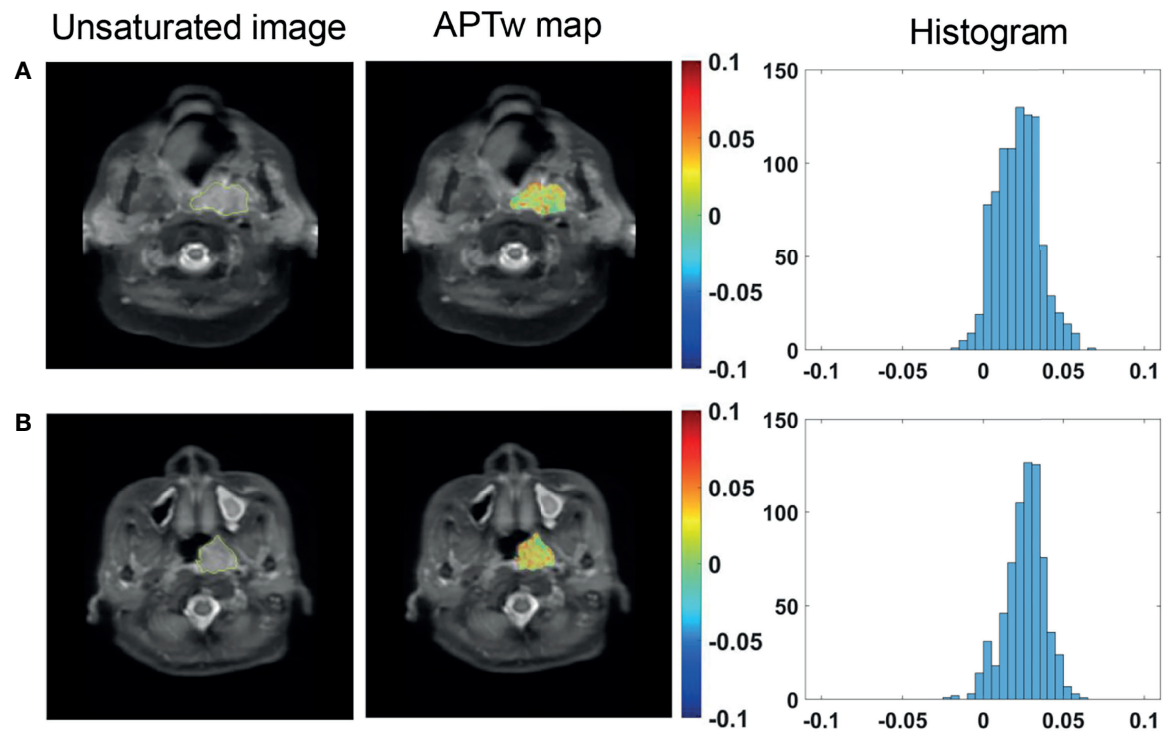

FIGURE 5 | Unsaturated SO image with ROI overlaid, APT $w_{w}$ maps and histograms of a 65-year-old male NPC patient with serum EBV-DNA (+) status (A), and a 47year-old female NPC patient with serum EBV-DNA (-) status (B).

TABLE 3 | APT-related parameters in groups with different EBV-related indices.

\begin{tabular}{|c|c|c|c|}
\hline & No. & $\mathrm{APT}_{\text {mean }}(\%)$ & $\mathrm{APT}_{90}(\%)$ \\
\hline \multicolumn{4}{|l|}{ EBV-DNA } \\
\hline+ & 23 & $1.72(1.48-2.04)$ & $3.44(3.12-4.04)$ \\
\hline Not available & 4 & - & - \\
\hline$P$-value & - & 0.995 & 0.730 \\
\hline \multicolumn{4}{|l|}{$E A-\lg A$} \\
\hline Not available & 46 & - & - \\
\hline$P$-value & - & 0.164 & 0.126 \\
\hline \multicolumn{4}{|l|}{ EB-VCA-IgA } \\
\hline+ & 27 & $1.72(1.59-1.74)$ & $3.05(2.92-3.38)$ \\
\hline- & 7 & $1.80(1.59-2.00)$ & $3.45(2.92-4.01)$ \\
\hline Not available & 46 & - & - \\
\hline$P$-value & - & 0.531 & 0.206 \\
\hline
\end{tabular}

show significant difference between responders and nonresponders. Such observations were consistent with our results that no significant difference in pretreatment $\mathrm{APT}_{\mathrm{w}}$ signals was presented among different treatment response groups. This might also imply that monitoring the dynamic protein change throughout the treatment course using $\mathrm{APT}_{\mathrm{w}}$ imaging may be more effective than detecting a stationary baseline pretreatment status in predicting treatment response.

Aside from the relatively limited sample size and imbalanced distribution in some groups, several limitations should be noted. First, conventional MTR asymmetry analysis was used to quantify APT effect, which would be contaminated with magnetization transfer and nuclear overhauser enhancement effects. However, the method has been histologically demonstrated to be applicable in tumor characterization (41). In addition, the $\mathrm{APT}_{\mathrm{w}}$ signal in tumor has proved mostly attributed to the APT contribution under the currently utilized RF power of $2 \mu \mathrm{T}$ (42). Therefore, the findings regarding APT are valid. Second, this study employed single-slice APT imaging to shorten the scan time, and the limited spatial coverage may be inadequate to characterize intra-tumoral heterogeneity. 3D APT scanning integrated with fast data sampling strategies would be desired for whole-tumor characterization in the future. 
TABLE 4 | Baseline APT-related parameters for different treatment response group.

\begin{tabular}{|c|c|c|c|}
\hline & No. & $\mathrm{APT}_{\text {mean }}(\%)$ & $\mathrm{APT}_{90}(\%)$ \\
\hline \multicolumn{4}{|c|}{ Overall treatment response } \\
\hline PD & 0 & - & - \\
\hline SD & 8 & $2.11(1.59-2.36)$ & $3.52(2.76-4.16$ \\
\hline PR & 41 & $1.77(1.50-2.04)$ & $3.39(3.13-3.74$ \\
\hline CR & 17 & $1.72(1.32-2.18)$ & $3.65(2.95-4.10$ \\
\hline$P$-value & - & 0.562 & 0.880 \\
\hline \multicolumn{4}{|c|}{ Overall treatment response based on SVRR } \\
\hline$<50 \%$ & 13 & $1.62(1.38-2.11)$ & $3.21(3.05-3.56$ \\
\hline$>50 \%$ & 53 & $1.80(1.48-2.16)$ & $3.45(3.13-4.04$ \\
\hline$P$-value & - & 0.514 & 0.306 \\
\hline \multicolumn{4}{|c|}{ Treatment response after induction chemotherapy } \\
\hline PD & 0 & - & - \\
\hline SD & 6 & $2.11(1.33-2.46)$ & $3.52(2.65-3.92$ \\
\hline PR & 35 & $1.77(1.45-2.01)$ & $3.25(3.10-3.78$ \\
\hline CR & 12 & $1.72(1.53-2.22)$ & $3.89(3.15-4.20$ \\
\hline$P$-value & - & 0.838 & 0.590 \\
\hline \multicolumn{4}{|c|}{ Treatment response after induction chemotherapy based on SVRR } \\
\hline$<50 \%$ & 11 & $1.53(1.33-2.01)$ & $3.21(3.01-3.57$ \\
\hline$>50 \%$ & 42 & $1.80(1.49-2.17)$ & $3.49(3.13-4.04$ \\
\hline$P$-value & - & 0.380 & 0.369 \\
\hline
\end{tabular}
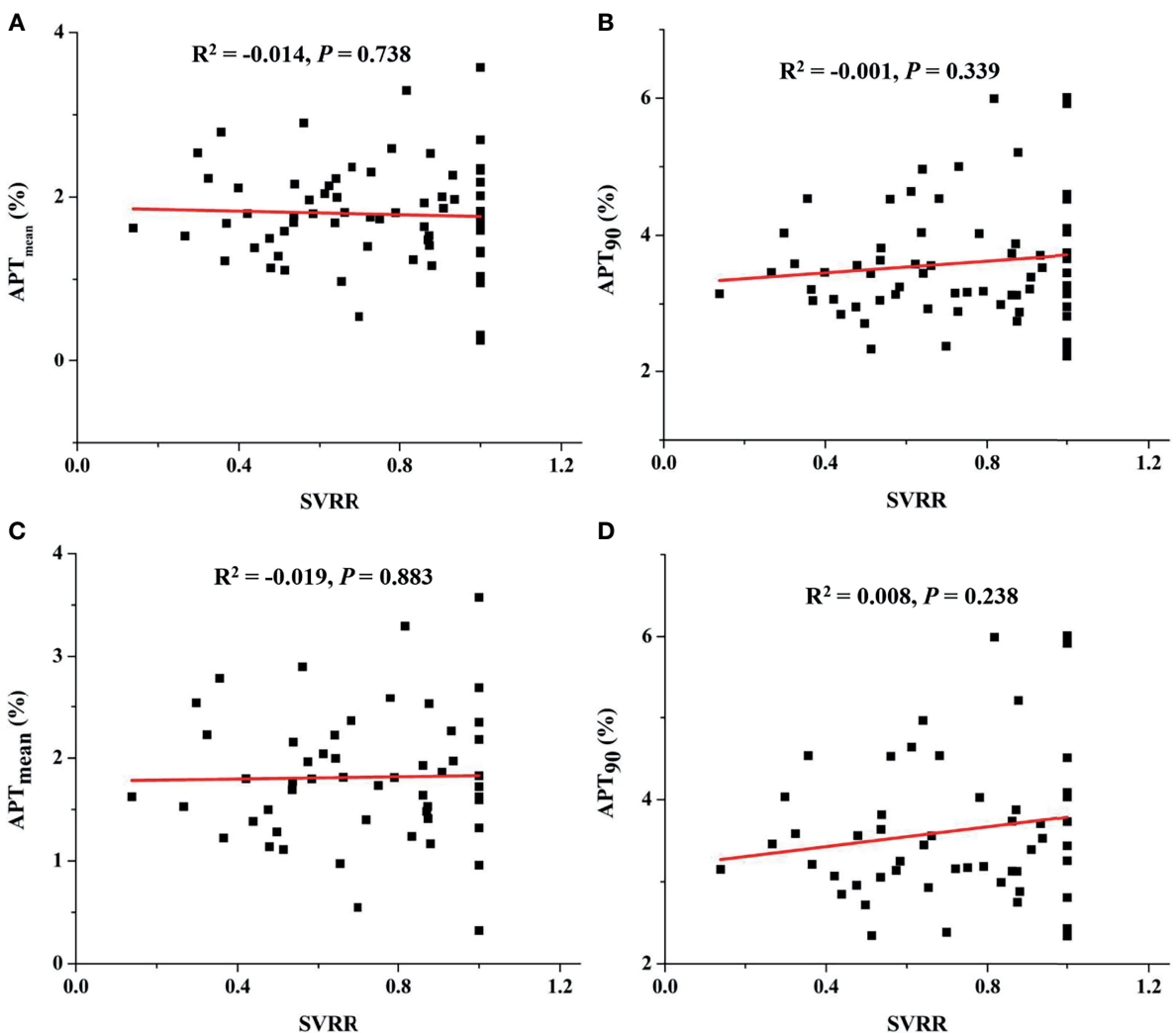

FIGURE 6 | For patients receiving overall treatment, correlation between $\mathrm{APT}_{\text {mean }}$ and SVRR (A), between APT 90 and SVRR (B), and for patients who received induction chemotherapy, correlation between APT mean and SVRR (C), between APT 90 and SVRR (D). 


\section{Conclusion}

In conclusion, NPC showed significantly stronger APT effect than normal nasopharyngeal tissue, facilitating NPClesion detection and early identification. However, baseline APT values showed limited value in predicting short-term response to IC and no significant correlation with different histopathological subtypes, EBV infection status, and clinical stages.

\section{DATA AVAILABILITY STATEMENT}

The original contributions presented in the study are included in the article/supplementary material. Further inquiries can be directed to the corresponding authors.

\section{ETHICS STATEMENT}

The studies involving human participants were reviewed and approved by the Cancer Hospital \& Shenzhen Hospital, Chinese Academy of Medical Sciences. Written informed consent for

\section{REFERENCES}

1. Bray F, Ferlay J, Soerjomataram I, Siegel RL, Torre LA, Jemal A. Global Cancer Statistics 2018: GLOBOCAN Estimates of Incidence and Mortality Worldwide for 36 Cancers in 185 Countries. CA Cancer J Clin (2018) 68 (6):394-424. doi: 10.3322/caac.21492

2. Wei KR, Zheng RS, Zhang SW, Liang ZH, Li ZM, Chen WQ. Nasopharyngeal Carcinoma Incidence and Mortality in China, 2013. Chin J Cancer (2017) 36 (1):90. doi: 10.1186/s40880-017-0257-9

3. Blanchard P, Lee A, Marguet S, Leclercq J, Ng WT, Ma J, et al. Chemotherapy and Radiotherapy in Nasopharyngeal Carcinoma: An Update of the MACNPC Meta-Analysis. Lancet Oncol (2015) 16(6):645-55. doi: 10.1016/s14702045(15)70126-9

4. Cao SM, Yang Q, Guo L, Mai HQ, Mo HY, Cao KJ, et al. Neoadjuvant Chemotherapy Followed by Concurrent Chemoradiotherapy Versus Concurrent Chemoradiotherapy Alone in Locoregionally Advanced Nasopharyngeal Carcinoma: A Phase III Multicentre Randomised Controlled Trial. Eur J Cancer (2017) 75:14-23. doi: 10.1016/j.ejca.2016.12.039

5. Peng H, Chen L, Li WF, Guo R, Mao YP, Zhang Y, et al. Tumor Response to Neoadjuvant Chemotherapy Predicts Long-Term Survival Outcomes in Patients With Locoregionally Advanced Nasopharyngeal Carcinoma: A Secondary Analysis of a Randomized Phase 3 Clinical Trial. Cancer (2017) 123(9):1643-52. doi: 10.1002/cncr.30520

6. Peng H, Chen L, Zhang Y, Li WF, Mao YP, Liu X, et al. The Tumour Response to Induction Chemotherapy has Prognostic Value for Long-Term Survival Outcomes After Intensity-Modulated Radiation Therapy in Nasopharyngeal Carcinoma. Sci Rep (2016) 6:24835. doi: 10.1038/srep24835

7. Xu J, Zhang K, Wang H, Cui Z, Wu Y, Wang W, et al. Clinical Application of Diffusion-Weighted Magnetic Resonance Imaging in Radiotherapy for Nasopharyngeal Carcinoma. J Xray Sci Technol (2019) 27(2):297-306. doi: 10.3233/xst-180441

8. Xiao Y, Chen Y, Chen Y, He Z, Yao Y, Pan J. Longitudinal Assessment of Intravoxel Incoherent Motion Diffusion Weighted Imaging in Evaluating the Radio-Sensitivity of Nasopharyngeal Carcinoma Treated With IntensityModulated Radiation Therapy. Cancer Res Treat (2019) 51(1):345-56. doi: $10.4143 /$ crt.2018.089 participation was not required for this study in accordance with the national legislation and the institutional requirements.

\section{AUTHOR CONTRIBUTIONS}

DL and YW contributed to project idea and supervision and to manuscript revision, and maintained integrity of this manuscript. ZL and LZ implemented the whole study, analyzed the data, and drafted the manuscript. QY and LQ contributed to statistical analysis and reviewed the manuscript. TL, HL, and CC collected the raw MRI data. YL collected the pathological data. PC and CQ collected clinical information. XL provided technical support. All authors listed have made a substantial, direct, and intellectual contribution to the work and approved it for publication.

\section{FUNDING}

The work described in this paper was supported by grants from the Key Areas Research and Development Program of Guangdong (2019B020235001) and the Shenzhen High-level Hospital Construction Fund.
9. Huang WY, Li MM, Lin SM, Chen F, Yang K, Zhu XL, et al. In Vivo Imaging Markers for Prediction of Radiotherapy Response in Patients With Nasopharyngeal Carcinoma: RESOLVE DWI Versus DKI. Sci Rep (2018) 8 (1):15861. doi: 10.1038/s41598-018-34072-9

10. Qin Y, Yu X, Hou J, Hu Y, Li F, Wen L, et al. Prognostic Value of the Pretreatment Primary Lesion Quantitative Dynamic Contrast-Enhanced Magnetic Resonance Imaging for Nasopharyngeal Carcinoma. Acad Radiol (2019) 26(11):1473-82. doi: 10.1016/j.acra.2019.01.021

11. Zhou J, Heo HY, Knutsson L, van Zijl PCM, Jiang S. APT-Weighted MRI: Techniques, Current Neuro Applications, and Challenging Issues. J Magn Reson Imaging (2019) 50(2):347-64. doi: 10.1002/jmri.26645

12. Meissner JE, Korzowski A, Regnery S, Goerke S, Breitling J, Floca RO, et al. Early Response Assessment of Glioma Patients to Definitive Chemoradiotherapy Using Chemical Exchange Saturation Transfer Imaging at 7 T. J Magn Reson Imaging (2019) 50(4):1268-77. doi: 10.1002/jmri.26702

13. Paech D, Dreher C, Regnery S, Meissner JE, Goerke S, Windschuh J, et al. Relaxation-Compensated Amide Proton Transfer (APT) MRI Signal Intensity is Associated With Survival and Progression in High-Grade Glioma Patients. Eur Radiol (2019) 29(9):4957-67. doi: 10.1007/s00330-019-06066-2

14. Park JE, Kim HS, Park KJ, Choi CG, Kim SJ. Histogram Analysis of Amide Proton Transfer Imaging to Identify Contrast-Enhancing Low-Grade Brain Tumor That Mimics High-Grade Tumor: Increased Accuracy of MR Perfusion. Radiology (2015) 277(1):151-61. doi: 10.1148/radiol.2015142347

15. Togao O, Hiwatashi A, Yamashita K, Kikuchi K, Keupp J, Yoshimoto K, et al. Grading Diffuse Gliomas Without Intense Contrast Enhancement by Amide Proton Transfer MR Imaging: Comparisons With Diffusion- and PerfusionWeighted Imaging. Eur Radiol (2017) 27(2):578-88. doi: 10.1007/s00330-0164328-0

16. Choi YS, Ahn SS, Lee SK, Chang JH, Kang SG, Kim SH, et al. Amide Proton Transfer Imaging to Discriminate Between Low- and High-Grade Gliomas: Added Value to Apparent Diffusion Coefficient and Relative Cerebral Blood Volume. Eur Radiol (2017) 27(8):3181-9. doi: 10.1007/s00330-017-4732-0

17. Ma B, Blakeley JO, Hong X, Zhang H, Jiang S, Blair L, et al. Applying Amide Proton Transfer-Weighted MRI to Distinguish Pseudoprogression From True Progression in Malignant Gliomas. J Magn Reson Imaging (2016) 44(2):45662. doi: $10.1002 / j m r i .25159$ 
18. Park KJ, Kim HS, Park JE, Shim WH, Kim SJ, Smith SA. Added Value of Amide Proton Transfer Imaging to Conventional and Perfusion MR Imaging for Evaluating the Treatment Response of Newly Diagnosed Glioblastoma. Eur Radiol (2016) 26(12):4390-403. doi: 10.1007/s00330-016-4261-2

19. Donahue MJ, Donahue PC, Rane S, Thompson CR, Strother MK, Scott AO, et al. Assessment of Lymphatic Impairment and Interstitial Protein Accumulation in Patients With Breast Cancer Treatment-Related Lymphedema Using CEST MRI. Magn Reson Med (2016) 75(1):345-55. doi: $10.1002 / \mathrm{mrm} .25649$

20. Takayama $Y$, Nishie A, Sugimoto M, Togao O, Asayama Y, Ishigami K, et al. Amide Proton Transfer (APT) Magnetic Resonance Imaging of Prostate Cancer: Comparison With Gleason Scores. Magma (2016) 29(4):671-9. doi: 10.1007/s10334-016-0537-4

21. Ohno Y, Yui M, Koyama H, Yoshikawa T, Seki S, Ueno Y, et al. Chemical Exchange Saturation Transfer MR Imaging: Preliminary Results for Differentiation of Malignant and Benign Thoracic Lesions. Radiology (2016) 279(2):578-89. doi: 10.1148/radiol.2015151161

22. Law BKH, King AD, Ai QY, Poon DMC, Chen W, Bhatia KS, et al. Head and Neck Tumors: Amide Proton Transfer MRI. Radiology (2018) 288(3):782-90. doi: 10.1148/radiol.2018171528

23. Yu L, Li C, Luo X, Zhou J, Zhang C, Zhang Y, et al. Differentiation of Malignant and Benign Head and Neck Tumors With Amide Proton TransferWeighted MR Imaging. Mol Imaging Biol (2019) 21(2):348-55. doi: 10.1007/ s11307-018-1248-1

24. Liu R, Jiang G, Gao P, Li G, Nie L, Yan J, et al. Non-Invasive Amide Proton Transfer Imaging and ZOOM Diffusion-Weighted Imaging in Differentiating Benign and Malignant Thyroid Micronodules. Front Endocrinol (Lausanne) (2018) 9:747. doi: 10.3389/fendo.2018.00747

25. Bae YJ, Choi BS, Jeong WJ, Jung YH, Park JH, Sunwoo L, et al. Amide Proton Transfer-Weighted MRI in the Diagnosis of Major Salivary Gland Tumors. Sci Rep (2019) 9(1):8349. doi: 10.1038/s41598-019-44820-0

26. Qamar S, King AD, Ai QY, Law BKH, Chan JSM, Poon DMC, et al. Amide Proton Transfer MRI Detects Early Changes in Nasopharyngeal Carcinoma: Providing a Potential Imaging Marker for Treatment Response. Eur Arch Otorhinolaryngol (2019) 276(2):505-12. doi: 10.1007/s00405-018-5231-x

27. Qamar S, King AD, Ai QH, Mo FKF, Chen W, Poon DMC, et al. PreTreatment Amide Proton Transfer Imaging Predicts Treatment Outcome in Nasopharyngeal Carcinoma. Eur Radiol (2020) 30(11):6339-47. doi: 10.1007/ s00330-020-06985-5

28. Lertbutsayanukul C, Kannarunimit D, Prayongrat A, Chakkabat C, Kitpanit S, Hansasuta P. Prognostic Value of Plasma EBV DNA for Nasopharyngeal Cancer Patients During Treatment With Intensity-Modulated Radiation Therapy and Concurrent Chemotherapy. Radiol Oncol (2018) 52(2):195203. doi: $10.2478 /$ raon-2018-0016

29. Wu SG, Lian CL, Wang J, Zhang WW, Sun JY, Lin Q, et al. The Effect of Histological Subtypes on Survival Outcome in Nasopharyngeal Carcinoma After Extensive Follow Up. Ann Transl Med (2019) 7(23):768. doi: 10.21037/ atm.2019.11.75

30. Huang SH, O'Sullivan B. Overview of the 8th Edition TNM Classification for Head and Neck Cancer. Curr Treat Options Oncol (2017) 18(7):40. doi: 10.1007/s11864-017-0484-y

31. Stancanello J, Terreno E, Castelli DD, Cabella C, Uggeri F, Aime S. Development and Validation of a Smoothing-Splines-Based Correction Method for Improving the Analysis of CEST-MR Images. Contrast Media Mol Imaging (2008) 3(4):136-49. doi: 10.1002/cmmi.240

32. Kim M, Gillen J, Landman BA, Zhou J, van Zijl PC. Water Saturation Shift Referencing (WASSR) for Chemical Exchange Saturation Transfer (CEST) Experiments. Magn Reson Med (2009) 61(6):1441-50. doi: 10.1002/ mrm.21873
33. Eisenhauer EA, Therasse P, Bogaerts J, Schwartz LH, Sargent D, Ford R, et al. New Response Evaluation Criteria in Solid Tumours: Revised RECIST Guideline (Version 1.1). Eur J Cancer (2009) 45(2):228-47. doi: 10.1016/ j.ejca.2008.10.026

34. Stelow EB, Wenig BM. Update From The 4th Edition of the World Health Organization Classification of Head and Neck Tumours: Nasopharynx. Head Neck Pathol (2017) 11(1):16-22. doi: 10.1007/s12105-017-0787-0

35. Yuan J, Chen S, King AD, Zhou J, Bhatia KS, Zhang Q, et al. Amide Proton Transfer-Weighted Imaging of the Head and Neck at 3 T: A Feasibility Study on Healthy Human Subjects and Patients With Head and Neck Cancer. NMR BioMed (2014) 27(10):1239-47. doi: 10.1002/nbm.3184

36. Chen Y, Li X, Song Y, Zhu X, Zhao J, Yan X, et al. The Diagnostic Efficacy of Amide Proton Transfer Imaging in Grading Gliomas and Predicting Tumor Proliferation. Neuroreport (2019) 30(2):139-44. doi: 10.1097/wnr. 0000000000001174

37. Wang HY, Chang YL, To KF, Hwang JS, Mai HQ, Feng YF, et al. A New Prognostic Histopathologic Classification of Nasopharyngeal Carcinoma. Chin J Cancer (2016) 35:41. doi: 10.1186/s40880-016-0103-5

38. Ng RH, Ngan R, Wei WI, Gullane PJ, Phillips J. Trans-Oral Brush Biopsies and Quantitative PCR for EBV DNA Detection and Screening of Nasopharyngeal Carcinoma. Otolaryngol Head Neck Surg (2014) 150 (4):602-9. doi: 10.1177/0194599813520136

39. Zhang Y, Tang LL, Li YQ, Liu X, Liu Q, Ma J. Spontaneous Remission of Residual Post-Therapy Plasma Epstein-Barr Virus DNA and Its Prognostic Implication in Nasopharyngeal Carcinoma: A Large-Scale, Big-Data Intelligence Platform-Based Analysis. Int J Cancer (2019) 144(9):2313-9. doi: $10.1002 / \mathrm{ijc} .32021$

40. Wang FH, Wei XL, Feng J, Li Q, Xu N, Hu XC, et al. Efficacy, Safety, and Correlative Biomarkers of Toripalimab in Previously Treated Recurrent or Metastatic Nasopharyngeal Carcinoma: A Phase II Clinical Trial (POLARIS02). J Clin Oncol (2021) 39(7):704-12. doi: 10.1200/jco.20.02712

41. Togao O, Yoshiura T, Keupp J, Hiwatashi A, Yamashita K, Kikuchi K, et al. Amide Proton Transfer Imaging of Adult Diffuse Gliomas: Correlation With Histopathological Grades. Neuro Oncol (2014) 16(3):441-8. doi: 10.1093/ neuonc/not158

42. Zhou J, Hong X, Zhao X, Gao JH, Yuan J. APT-Weighted and NOE-Weighted Image Contrasts in Glioma With Different RF Saturation Powers Based on Magnetization Transfer Ratio Asymmetry Analyses. Magn Reson Med (2013) 70(2):320-7. doi: 10.1002/mrm.24784

\section{Conflict of Interest: Author LQ was employed by GE Healthcare.}

The remaining authors declare that the research was conducted in the absence of any commercial or financial relationships that could be construed as a potential conflict of interest.

Publisher's Note: All claims expressed in this article are solely those of the authors and do not necessarily represent those of their affiliated organizations, or those of the publisher, the editors and the reviewers. Any product that may be evaluated in this article, or claim that may be made by its manufacturer, is not guaranteed or endorsed by the publisher.

Copyright (c) 2022 Liu, Zou, Yang, Qian, Li, Luo, Che, Lei, Chen, Qiu, Liu, Wu and Luo. This is an open-access article distributed under the terms of the Creative Commons Attribution License (CC BY). The use, distribution or reproduction in other forums is permitted, provided the original author(s) and the copyright owner(s) are credited and that the original publication in this journal is cited, in accordance with accepted academic practice. No use, distribution or reproduction is permitted which does not comply with these terms. 